\title{
Diverse Roles of F-BoxProtein3 in Regulation of Various Cellular Functions
}

\author{
Zhiyang Zhang ${ }^{1 \dagger}$, Zhengqi Bao ${ }^{2 \dagger}$, Penglian Gao ${ }^{1}$, Junyi Yao ${ }^{1}$, Peter Wang ${ }^{3 *}$ and \\ Damin Chai ${ }^{1 *}$
}

${ }^{1}$ Department of Pathology, The First Affiliated Hospital of Bengbu Medical University, Bengbu, China, ${ }^{2}$ Department of Orthopedics, The First Affiliated Hospital of Bengbu Medical University, Bengbu, China, ${ }^{3}$ Bengbu Medical College Key Laboratory of Cancer Research and Clinical Laboratory Diagnosis, Bengbu Medical College, Bengbu, China

OPEN ACCESS

Edited by:

Chandrima Das,

Saha Institute of Nuclear Physics

(SINP), India

Reviewed by:

Heike Laman,

University of Cambridge,

United Kingdom

Han Shi,

Northwestern University,

United States

Xiangming $\mathrm{Ji}$,

Georgia State University,

United States

*Correspondence:

Peter Wang

wangpeter3@126.com

Damin Chai

chaidm2020@163.com

${ }^{\dagger}$ These authors have contributed equally to this work

Specialty section:

This article was submitted to

Epigenomics and Epigenetics,

a section of the journal

Frontiers in Cell and Developmental

Biology

Received: 26 October 2021

Accepted: 23 December 2021

Published: 19 January 2022

Citation:

Zhang Z, Bao Z, Gao P, Yao J, Wang P and Chai $D$ (2022) Diverse Roles of $F$ -

BoxProtein3 in Regulation of Various

Cellular Functions.

Front. Cell Dev. Biol. 9:802204.

doi: 10.3389/fcell.2021.802204
Accumulated evidence shows that the F-box protein 3 (FBXO3) has multiple biological functions, including regulation of immune pathologies, neuropathic diseases and antiviral response. In this review article, we focus on the role of $\mathrm{FBXO} 3$ in inflammatory disorders and human malignancies. We also describe the substrates of FBXO3, which contribute to inflammatory disorders and cancers. We highlight that the high expression of FBXO3 is frequently observed in rheumatoid arthritis, leukemia, pituitary adenoma, and oral squamous cell carcinoma. Moreover, we discuss the regulation of FBXO3 by both carcinogens and cancer preventive agents. Our review provides a comprehensive understanding of the role of $\mathrm{FBXO} 3$ in various biological systems and elucidates how FBXO3 regulates substrate ubiquitination and degradation during various physiological and pathological processes. Therefore, $\mathrm{FBXO} 3$ can be a novel target in the treatment of human diseases including carcinomas.

Keywords: Fbxo3, carcinomas, inflammation, rheumatoid arthritis, ubiquitination

\section{INTRODUCTION}

Ubiquitination is one type of post-translational modifications (PTMs), which regulates cellular protein concentrations in eukaryotic organisms (Senft et al., 2018). The ubiquitin protease system selectively targets multiple proteins for degradation through the use of activating (E1), conjugating (E2) and ligating (E3) enzymes (Popovic et al., 2014). In particular, E3 ubiquitin ligases determine substrate specificity for ubiquitination and then transfer ubiquitin chains to the substrate, which leads to substrate degradation in the $26 \mathrm{~S}$ proteasome (Grabbe et al., 2011). Ubiquitination is involved in the regulation of almost all cellular activities, including embryonic development, cell proliferation, apoptosis, autophagy, signal transduction and DNA repair (Grabbe et al., 2011; Popovic et al., 2014). In recent years, Bortezomib as a proteasome inhibitor has been approved for the treatment of multiple myeloma and mantle cell lymphoma (Palumbo et al., 2016). Targeting specific substrates for ubiquitination has become a new clinical therapeutic strategy.

The ubiquitin protein ligase complex Skp1-Cullin1-F-Box (SCF) is composed of four subunits: F-box protein, SKP1, CULLIN1, and RBX1 (Skaar et al., 2014). Twenty years after the discovery of the F-box protein family, around 70 different F-box proteins have now been identified in mammals (Skaar et al., 2013). F-box proteins are classified into three categories based on the type of C-terminal interaction: FBXW (containing WD40 repeats), FBXL (containing leucine-rich repeats), and FBXO (containing neither, but with other domains) (Wang et al., 2014). The F-box protein directs the SCF complex to specific substrates for ubiquitination. Emerging evidence has demonstrated that F-box 
proteins are associated with the aggressiveness of human tumors, cell cycle regulation, and regulation of the epithelialmesenchymal transition (EMT) and cancer stem cells (CSCs) as well as drug resistance (Song et al., 2019; Yan et al., 2020). The F-box protein 3 (FBXO3), also known as FBX3, F-box only protein 3, F-box protein FBX3, and FBX, is encoded by a gene located on chromosome 11p13. Human FBXO3 gene has 13 splicing variants and belongs to the F-box protein family. Recently, studies have shown that FBXO3 participates in immune pathologies, neuropathic diseases, antiviral response, inflammatory disorders and human malignancies (Mallampalli et al., 2013; Lin et al., 2015; Shao et al., 2016; Hung et al., 2019). In the following paragraphs, we describe how $\mathrm{FBXO} 3$ contributes to inflammatory disorders and cancers, including leukemia, pituitary adenoma, oral squamous cell carcinoma and breast cancer. Moreover, we describe the regulatory mechanism of FBXO3 by carcinogens and cancer preventive agents.

\section{Structure of FBXO3}

The human $\mathrm{FBXO} 3$ protein has 471 amino acids, with a molecular mass of $54,561 \mathrm{Da}$. Alternative splicing of FBXO3 gene generates two transcript variants diverging at the $3^{\prime}$ end. FBXO3 has two domains at its C-terminal: F-box domain (positions 10-56) and ApaG (Adenine tetraphosphate adenine $\mathrm{G}$ ) domain (positions 278-408). ApaG domain is involved in mediating the ubiquitination and degradation of F-box and leucine-rich repeat protein 2 (FBXL2), resulting in cytokine gene transcription and promoting the progression of inflammation (Mallampalli et al., 2013). The relevant ApaG domain consists of an immunoglobin/fibronectin III-type fold and a classical $\beta$-sheet core. The central $\beta$-sheet core is a potential target in drug discovery, which aims at regulating inflammation and malignancies (Krzysiak et al., 2016). The FBXO3 has three described isoforms: isoform 1 (Q9UK99-1), isoform 2 (Q9UK992, 414-415: EM $\rightarrow \mathrm{VS}$, 416-471: Missing), and isoform 3 (Q9UK99-3, 36-40: Missing, 414-415: EM $\rightarrow$ VS, 416-471: Missing). The FBXO3 protein recognizes specific substrates for ubiquitination and degradation. Additional structural studies are needed to elucidate the functions of each domain of the FBXO3 protein in its various physiological and pathological processes.

\section{FBXO3 Regulates Inflammation}

Following infection with a virulent pathogen, there is an excessive release of cytokines from proinflammatory cells, including macrophages, lymphocytes and polymorpho nuclear leukocytes (Dinarello, 2007; Sheu et al., 2010). This process, known as a "cytokine storm", leads to hypercytokinemia where in hypercytokines it increases capillary permeability and tissue edema causing fever, pain, multiple organ failure, and even death (Nathan, 2002; Aird, 2003). TRAF proteins are cytokine signaling adapter proteins that are critically involved in inflammation and programmed cell death (Inoue et al., 2000; Mallampalli et al., 2013). FBXO3 proteins are critically involved in inflammation and target FBXL2 for degradation, partly promoting TNF receptor-associated factor (TRAF) signal transduction and cytokine gene transcription (Inoue et al., 2000; Mallampalli et al., 2013). Chen et al. reported that mice with FBXO3 knockout that are infected with Pseudomonas aeruginosa showed reduced lavage cytokine levels, protein concentrations, and proinflammatory cell counts in the lung tissue. This suggests that FBXO3 knockdown attenuates lung injury induced by Pseudomonas and reduces mortality (Chen et al., 2013). Typically, patients with sepsis die of organ dysfunction because of an unusually strong reaction response to an infection (Pantzaris et al., 2021). The researchers also found that subjects with sepsis had more TRAF and FBXO3 proteins and less FBXL2 protein in circulating white blood cells compared with control subjects. Moreover, the circulating FBXO3 and TRAF proteins in sepsis patients had positive correlations with cytokine responses (Chen et al., 2013).

Studies of the FBXO3 C-terminal structure demonstrate that the classical ApaG molecular features are indispensable for mediating FBXL2 ubiquitination and for promoting the release of cytokines (Krzysiak et al., 2016). This leads to the development of BC- 1215 compound, which is a highly selective small molecule as a FBXO3 antagonist targeting the ApaG domain (Chen et al., 2013). BC-1215 decreases FBXO3-FBXL2 interaction in a dosedependent manner and protects FBXL2 from FBXO3-induced degradation, which effectively lowers the expression of TRAF1TRAF6 proteins. The addition of $\mathrm{BC}-1215$ reduces proinflammatory cytokines and modestly inhibits bacterial growth in a mouse model of cecal ligation and perforation to induce sepsis (Chen et al., 2013). Treatment with BC-1215 or knockdown of FBXO3 were found to attenuate the inflammation of lung tissue induced by Pseudomonas and the H1N1 influenza virus, ear injury induced by tetradecanoylphorbol, and active colitis induced by acetate dextran sulfate sodium (Chen et al., 2013; Mallampalli et al., 2013). Furthermore, the downregulation of FBXO3 levels attenuates lung edema induced by ischemiareperfusion (I/R) (Hung et al., 2019).

Oxygen glucose deprivation/re-oxygenation (OGD/R) model is often used for ischemia studies via oxygen and glucose deprivation and then reoxygenation to mimic ischemia/ reperfusion injury condition. In the OGD/R model, miR-142$3 \mathrm{p}$ directly targets $\mathrm{FBXO} 3$ to ameliorate inflammation and apoptosis in SH-SY5Y cells (Li and Ma, 2020). The inflammasome is a complex composed of multiple proteins that regulate the maturation and release of pro-inflammatory cytokines, such as IL-1 $\beta$ and IL-18. Lipopolysaccharide (LPS) exposure attenuates FBXL2-induced NALP3 inflammasome ubiquitination by activating $\mathrm{FBXO}$, thereby increasing the secretion of IL-1 $\beta$ and IL-18 in inflammatory cells (Han et al., 2015). FBXO3 also potentiates vascular inflammation and increases atherosclerosis. Depletion of FBXO3 protein in macrophages eliminates oxidatively modified low-density lipoprotein-induced inflammation without affecting oxidized low-density lipoprotein uptake by macrophages (Chandra et al., 2019). Treatment with BC-1215 reduces the release of IL- $1 \beta$ and TNF- $\alpha$ (Chandra et al., 2019), which alleviates FBXO3induced vascular inflammation and atherosclerosis. These data suggest that FBXO3 is a novel target of drug design that aims to alleviate atherosclerosis driven by pro-inflammatory cytokines. This benefit might extend beyond low-density lipoprotein reduction. 


\section{FBXO3 Regulates Neuropathic Pain}

Rab3-interactive molecule-1a (RIM1a) is essential for C-terminal regions-associated vesicle exocytosis (Schoch et al., 2006) and spinal plasticity in the presynaptic site of the dorsal horn, which contributes to the development of neuropathic pain. The voltagegated $\mathrm{N}$-type $\mathrm{Ca}^{2+}$ channel (Cav2.2) has been demonstrated that promoted neuropathic pain in mice model (Saegusa and Tanabe, 2014). Besides, it has been reported that RIM is related to Cav2.2 in neuropathic pain via promotion of vesicle exocytosis (Coppola et al., 2001; Hibino et al., 2002). One study demonstrated that FBXO3-dependent FBXL2 ubiquitination promotes RIM1a/ CaV2.2 cascade in neuropathic pain based on spinal plasticity (Lin et al., 2015). FBXO3 degrades FBXL2 and leads to deubiquitination of RIM1, resulting in enhanced RIM1 interaction with the $\mathrm{CaV} 2.2$, which contributes to chronic pain due to upregulating CaV2.2 (Lai et al., 2016).

The roles of NcK-interacting kinase (TNIK) in neuropathic pain development is coupling TNIK-GluR1 and leads to subcellular redistribution of GluR1-AMPA receptors (AMPARs) (Hussain et al., 2010). TRAF2 enhances TNIK/GluR1 phosphorylationdependent subcellular GluR1-AMPARs redistribution, leading to spinal nerve ligation-induced allodynia (Lin et al., 2015). Allodynia is a kind of pain due to a hypersensitive reaction to a normal stimulus. Similar to RIM1a, TRAF2 is also regulated by FBXO3-dependent FBXL2 (Chen et al., 2013; Mallampalli et al., 2013). FBXO3 is involved in neuropathic allodynia via its effects on degradation of FBXL2 and upregulation of TRAF2, and administration of BC-1215 ameliorates this allodynia (Lin et al., 2015). While the research in this field is still infancy, FBXO3 might provide a potential drug target for neuropathic pain relief.

\section{FBXO3 Regulates Autoimmunity Functions}

Autoimmune regulator (AIRE) as a transcription factor is crucial for the maintenance of self-tolerance (Finnish-German, 1997; Nagamine et al., 1997). Impairment of AIRE activity is implicated in disturbed negative selection of $\mathrm{T}$ cells that are specific for selfantigens, which leads to lymphocytic infiltration of affected organs and causes disorders in immunological homeostasis. These ultimately result in autoimmune diseases, including type 1 diabetes mellitus, thymomas, and autoimmune thyroid diseases (Peterson and Peltonen, 2005; Sabater et al., 2005; Perheentupa, 2006; Gavanescu et al., 2007). FBXO3 has been reported to regulate autoimmunity by promoting the ubiquitination and transcriptional activity of the AIRE (Shao et al., 2016). AIRE is phosphorylated on the Thr-68 and Ser-156 residues near its $\mathrm{N}$-terminus allowing it to bind to $\mathrm{FBXO} 3$ and becomes ubiquitinated. The ubiquitination of AIRE increases the activity of tissue-specific antigens (TSA) genes and enhances the recruitment of positive transcription elongation factor $b$ (P-TEFb) to target genes (Shao et al., 2016), which influences the maturation of thymocytes (Peterson et al., 2008).

\section{FBXO3 Negatively Regulates Antiviral Response}

Rift Valley fever virus (RVFV) infection can cause animal-derived diseases, which are transmitted to people mainly through mosquito bites or contact with infected livestock (Bird et al., 2009; Boshra et al., 2011). RVFV is prevalent in Africa and has caused frequent outbreaks that result in devastating loss of lives and properties. It is known that interferon activation is essential for antiviral response. One group showed that FBXO3 inhibits the antiviral response in host cells. As the main virulence factor of RVFV, the nonstructural protein NSs recruits FBXO3 to degrade the transcription factor TFIIH-p62 of host cells (Kainulainen et al., 2014). This facilitates the pathogenesis of RVFV by inhibiting transcriptional upregulation of the innate immunity and hindering the antiviral type I interferon (IFN-I) system to allow uncontrolled viral replication (Bouloy et al., 2001; Billecocq et al., 2004). NSs interacts with the full-length FBXO3 as well as with a truncated isoform that lacks the C-terminal acidic and poly(R)-rich domains (Kainulainen et al., 2014).

The genome structures of type 1 IFN in fish are similar with mammals (Kirsten et al., 2018). IRF3 and IRF7 are transcription factors of the IFN regulatory factor (IRF) family that induce IFN expression ( $\mathrm{Lu}$ et al., 2019). IFN induces transcription of downstream antiviral genes through activation of JAK-STAT signaling pathway (Cheng et al., 2019). IRF3 and IRF7 degradation and IFN signaling activation are critical for FBXO3-mediated antiviral response in zebrafish. FBXO3 negatively regulates antiviral response by promoting K27linked ubiquitination and proteasomal degradation of IRF3 and IRF7 in an F-box domain-independent manner (Li et al., 2020). In zebrafish model, FBXO3 deletion induces the expression of key antiviral genes and shows higher resistance to virus infection in liver and spleen (Li et al., 2020).

\section{Role of FBXO3 in Rheumatoid Arthritis}

Rheumatoid arthritis (RA) is an autoimmune disease because immune system hurts healthy cells, leading to erosion of bone and cartilage in joints, especially in the hands, knees and wrists, which causes pain, stiffness, swelling and dysfunctions in the joints (Wang et al., 2019). To investigate the role of FBXO3 in RA, Masuda et al. examined the expression of a diverse array of genes through in situ hybridization (Masuda et al., 2002). They concluded that some proliferation-related molecules, including FBXO3, displayed higher expression levels in RA synovial tissues compared to normal synovial tissues (Masuda et al., 2002). The involvement of FBXO3 in RA has not been clearly demonstrated. It is known that F-box proteins regulate the cell cycle (Winston et al., 1999), FBXO3 might be potentially involved in survival and proliferation of RA synovial fibroblast (RA-SF).

\section{Role of FBXO3 in Amyotrophic Lateral Sclerosis}

Amyotrophic lateral sclerosis (ALS) has a poor prognosis, and most of patients with ALS die within 3-7 years after involving the respiratory muscles (Talbott et al., 2016). The pathogenic mechanism is still unclear, and no effective treatment is available. Chromosome 21 open reading frame 2 (C21ORF2) interacts with never in mitosis gene A related kinase 1 (NEK1) that involve in DNA damage repair and regulate cell cycle, which is highly related to ALS development (Fang et al., 2015; Chia et al., 
2018). Watanabe et al. showed that Fbxo3 targeted C21ORF2 for ubiquitination and degradation (Watanabe et al., 2020). Because NEK1 was stabilized by C21ORF2, depletion of FBXO3 stabilized both C21ORF2 and NEK1. NEK1-mediated phosphorylation of C21ORF2 can protect it from proteasome-dependent degradation due to attenuating the interaction between FBXO3 and C21ORF2 (Watanabe et al., 2020). Inhibition of NEK1 activity and increased degradation of C21ORF2 by FBXO3 may be potential approaches for treatment of patients with ALS.

\section{Role of FBXO3 in Human Cancers FBXO3 Regulates Pathogenesis in Several Types of Cancers}

FBXO3 has been demonstrated to participate in the occurrence and progression of a variety of human cancers. It is noteworthy that in acute promyelocytic leukemia, the PML gene is the site of the $t(15$, 17) chromosomal translocation wherein it is fused to the retinoic acid receptor (RAR) gene, leading to the generation of PML-RAR fusion protein (de Thé et al., 1990; de Thé et al., 1991; Goddard et al., 1991; Kakizuka et al., 1991). It has been shown that PML activates transcription by preventing FBXO3-catalyzed ubiquitination of HIPK and p300 (Shima et al., 2008). Conversely, PML-RAR enhances FBXO3-induced degradation of HIPK2 and p300 in a dose-dependent manner to inhibit transcription, which might contribute to the pathogenesis of leukemia (Shima et al., 2008). Another study by Laura et al. analyzed the relationship between FBXO3 and pituitary adenoma and showed that FBXO3 enhances the degradation of the aryl hydrocarbon receptor-interacting protein (AIP) through the rapid ubiquitin proteasome pathway, which has direct implications for the phenotype. Through this, a novel pathogenic mechanism of pituitary adenoma was generated (Hernández-Ramírez et al., 2016). In a work done by Cha et al., which employed a combination of array-based comparative genomic hybridization and multiplex ligation-dependent probe amplification, it was found that the highest deletion frequencies in $\mathrm{FBXO} 3$ might be related to the occurrence and progression of oral squamous cell carcinoma (Cha et al., 2011).

The FBXO3- $\triangle \mathrm{Np} 63 \alpha$ is critical for TGF- $\beta$-induced tumor metastasis (Hao et al., 2019). $\Delta \mathrm{Np} 63$ has been identified as a subtype of p63 that leads to regulation of cell proliferation, cell adhesion, EMT, and inhibition of tumor metastasis (Bergholz and Xiao, 2012). FBXO3 promotes breast cancer metastasis through K48-linked polyubiquitination of the $\Delta \mathrm{Np} 63 a$. This process is independent of Smad but dependent of Erk (Niu et al., 2021). Upregulation of FBXO3 by activation of TGF- $\beta$ results in the degradation of $\triangle \mathrm{Np} 63 \alpha$ with concomitant decreased expression of E-cadherin and desmoplakin (DPL) (Niu et al., 2021). In addition, the high expression of $\mathrm{FBXO} 3$ indicates poor prognosis in patients with breast cancer (Niu et al., 2021). Taken together, FBXO3 targets different substrates to participate in carcinogenesis.

\section{FBXO3 Regulates Smurf1 and BMP Pathway}

The Smad ubiquitination regulatory factor 1 (Smurf1) is a member of the HECT-type E3 ubiquitin ligases and based on its C2-WW-HECT architecture, which belongs to the neural precursor cell-expressed and developmentally downregulated gene 4 (Nedd4) family of lipases (Fu et al., 2020; Wang et al., 2020). Smurf1 regulates several biological pathways, including the transforming growth factor beta (TGF- $\beta$ ), the bone morphogenetic protein (BMP), the non-canonical Wnt pathway, and the mitogen-activated protein kinase (MAPK) pathway (Fu et al., 2020). Smurf1 is also related to cell growth and migration, embryonic development, immune responses, and tumorigenesis. FBXO3 targets all the Nedd4 family members including Smurf1 for degradation (Li et al., 2015). FBXO3 upregulates BMP pathway by mediating Smurf1 ubiquitination in vivo as well as in vitro and $\mathrm{FBXO} 3$ significantly promotes the poly-ubiquitination of Smurf1 (Li et al., 2015).

\section{FBXO3 Regulates DNA Damage}

The mouse homolog of diaphanous 2 (mDia2) belongs to the diaphanous-related formin 1 (mDia1) family. mDia2 influences the remodeling of actin and microtubule cytoskeletons after transformation to its active conformation (Chesarone et al., 2010), and plays a crucial role in cell invasion and cytokinesis (Lizárraga et al., 2009; Daou et al., 2014). FBXO3 forms a complex with mDia2 and p53, and co-expression of mDia2 and FBXO3 promotes p53-dependent apoptosis in an actin-nucleationindependent manner (Isogai et al., 2015). As a tumor suppressor, p53 regulates cell growth through cellular apoptotic programs and DNA repair. FBXO3 knockdown attenuates p53-mediated apoptosis upon DNA damage (Bieging and Attardi, 2012).

\section{FBXO3 Regulates Cell Apoptosis}

Studies have shown that FBXO3 contributes to tumor progression but also increases tumor cell apoptosis. Recruitment of histone deacetylases by oncoproteins is a key inciting event for cancer progression (Hess-Stumpp, 2005; Minucci and Pelicci, 2006). In lung squamous cell carcinoma, Kong et al. demonstrated that treatment with the histone deacetylase inhibitor belinostat (PXD101) transcriptionally upregulates FBXO3 and FBXW10, which directly target son of sevenless (SOS), an upstream regulator of the MAPK pathway, to inhibit growth of tumor cells (Kong et al., 2017). Thus, the induction of tumor cell apoptosis is increased, and drug resistance to cisplatin is reduced. This suggests that targeting FBXO3 might be a novel strategy for cancer treatment.

\section{FBXO3 Is Regulates by miRNAs}

MicroRNAs (miRNAs) are small non-coding RNAs that regulate target genes at the post-transcriptional level (Winkle et al., 2021). Notably, non-coding RNAs are essential for maintaining cellular homeostasis and perform their functions by regulating cell proliferation, migration, invasion, metastasis, and apoptosis (Goodall and Wickramasinghe, 2021; Jiang et al., 2020). The levels of some miRNAs have been shown to correlate with various cancers through negatively regulating genes including F-box proteins (Lin et al., 2019). Analyses using the TargetScan online computational algorithm (www.targetscan.org) and luciferase reporter genes showed that FBXO3 is identified as a 
TABLE 1 | The roles of FBXO3 in various biological functions.

Functions

Regulates inflammation

Neuropathic pain

Immunoregulation

Negatively regulates antiviral response

Rheumatoid arthritis

Leukemia

Pituitary adenoma

Oral squamous cell carcinoma

Breast cancer

Tumorigenesis

\section{Targets}

FBXL2

FBXL2

AIRE

TFIIH-p62

N/A

HIPK, p300

AIP

N/A

$\Delta$ Np63

Smurf1
References

Mallampalli et al. (2013)

Lin et al. (2015)

Shao et al. (2016)

Kainulainen et al. (2014)

Masuda et al. (2002)

Shima et al. (2008)

Hernández-Ramírez et al. (2016)

Cha et al. (2011)

Niu et al. (2021)

Li et al. (2015)

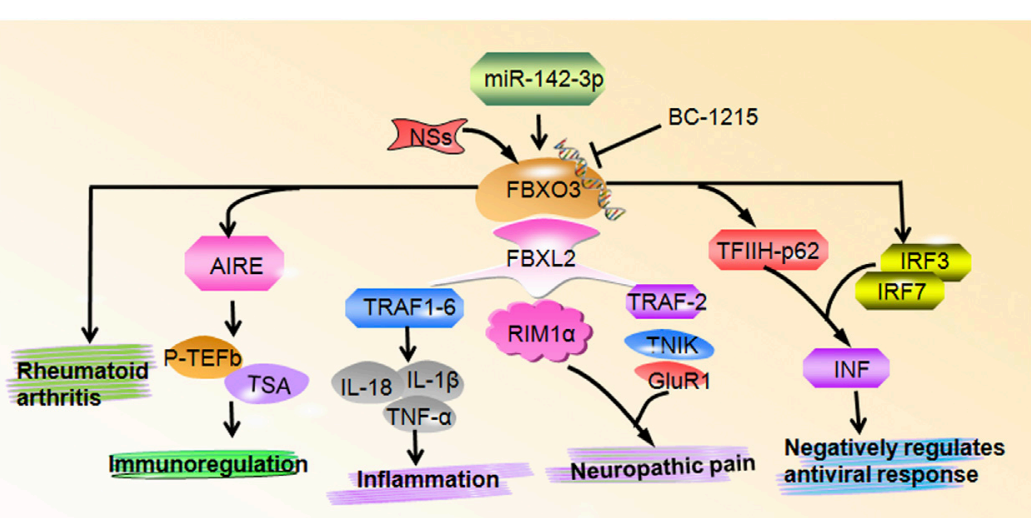

FIGURE 1 | FBXO3 regulates multiple biological functions. FBXO3 controls numerous cellular signaling pathways and genes to participate in inflammation, neuropathic pain, immunoregulation, negatively regulates antiviral response and rheumatoid arthritis.

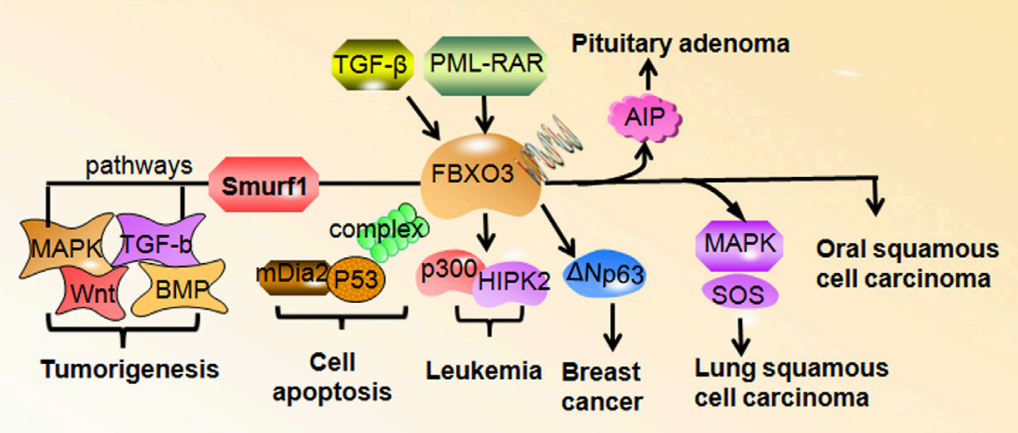

FIGURE 2 |FBXO3 regulates downstream targets in human cancers. FBXO3 targets numerous genes to participate in tumorigenesis and cell apoptosis. FBXO3 is regulated by TGF-ßand PML-RAR. The high expression of FBXO3 is frequent in rheumatoid arthritis, leukemia, pituitary adenoma, oral squamous cell carcinoma.

target of miR-142-3p (Li and $\mathrm{Ma}, 2020)$. It has been shown that miR-142-3p plays key roles in tumorigenesis and cancer progression and is expressed at lower levels in breast tumor tissues than in those from normal individuals (Xu et al., 2020). miR-142-3p negatively regulates the canonical Wnt signaling pathway to regulate human breast cancer stem cells (Isobe et al., 2014). Several studies have also found that miR-142-3p is an important regulatory element in hepatocellular carcinoma, cervical cancer, non-small cell lung carcinoma, and glioblastoma (Chai et al., 2014; Xiao and Liu, 2015; Shrestha et al., 2017; Dong and Song, 2021). Furthermore, miR-142-3p has also been linked to inhibition of tumor progression and invasion. Thus, miR-142$3 p$ might be useful in targeting cancer stem cells (Ghafouri-Fard et al., 2021). However, the relation between miR-142-3p to 
FBXO3 in human cancer cells is not yet clear and needs further research.

\section{CONCLUSION AND PERSPECTIVE}

FBXO3 is involved in numerous biological functions and has an important impact on promoting inflammation, immune regulation, the inhibition of IFN-I that triggers virus replication, and the processes of neuropathic pain and rheumatoid arthritis (Table 1 and Figure 1). Among these, the most studies determine the role of $\mathrm{FBXO} 3$ in the pathophysiological mechanism of inflammation. According to previous researches, $\mathrm{FBXO} 3$ is a critical modulator of inflammation which can inhibit LPS stimulation of inflammatory responses by promoting and inhibiting the degradation of FBXL2 and TRAFs, respectively. A new area being explored in cancer research is the role of ubiquitination in inflammasome biology. Leucine-rich repeat receptors (NLRs) and melanoma 2 (AIM2)-like receptors (ALRs) families are important in the assembly of the inflammasome complex (Schroder and Tschopp, 2010). Among these, NLRP1, NLRP3, and NLRC4 are linked to inflammatory diseases and colorectal cancer (Jin et al., 2007; Man and Kanneganti, 2015). The formulation of new immunotherapy to regulate inflammasomes governed by ubiquitination can provide a novel strategy for the treatment of diseases.

A review of literature shows contradicting reports on the effect of FBXO3 on tumor development (Figure 2). The expression of FBXO3 is increased in oral squamous cell carcinoma, acute promyelocytic leukemia, pituitary adenoma, and breast cancer. However, co-expression of $\mathrm{FBXO} 3$ and $\mathrm{p} 53$ promotes apoptosis of tumor cells (Bieging and Attardi, 2012). In addition, FBXO3 in combination with chemotherapeutic drugs can reduce drug resistance and increase chemical sensitization. These independent findings corroborate the potential roles of FBXO3 in the processes of anti-tumor activity and progression of tumors (Kong et al., 2017). While this may complicate the treatment, the specificity of FBXO3 makes it an attractive therapeutic target.

\section{REFERENCES}

Aird, W. C. (2003). The Role of the Endothelium in Severe Sepsis and Multiple Organ Dysfunction Syndrome. Blood 101, 3765-3777. doi:10.1182/blood-200206-1887

Bergholz, J., and Xiao, Z.-X. (2012). Role of P63 in Development, Tumorigenesis and Cancer Progression. Cancer Microenvironment 5, 311-322. doi:10.1007/ s12307-012-0116-9

Bieging, K. T., and Attardi, L. D. (2012). Deconstructing P53 Transcriptional Networks in Tumor Suppression. Trends Cel Biol. 22, 97-106. doi:10.1016/ j.tcb.2011.10.006

Billecocq, A., Spiegel, M., Vialat, P., Kohl, A., Weber, F., Bouloy, M., et al. (2004). NSs Protein of Rift Valley Fever Virus Blocks Interferon Production by Inhibiting Host Gene Transcription. J. Virol. 78, 9798-9806. doi:10.1128/ jvi.78.18.9798-9806.2004

Bird, B. H., Ksiazek, T. G., Nichol, S. T., and Maclachlan, N. J. (2009). Rift Valley Fever Virus. J. Am. Vet. Med. Assoc. 234, 883-893. doi:10.2460/javma.234.7.883
However, the association of the expression of $\mathrm{FBXO} 3$ with tumor size, tumor stage, deep of infiltration, and prognosis in cancer patients has not yet been well established. The specific mechanism is also not clear, and there is still a lack of clinical data on FBXO3-related tumors.

In order to fully understand the role of $\mathrm{FBXO} 3$ in tumorigenesis, the following questions need to be addressed. What are the expression levels of FBXO3 in many human cancers other than oral squamous cell carcinoma, acute promyelocytic leukemia, and pituitary adenoma? What are the carcinogenic or anticancer signaling pathways that trigger FBXO3-induced oncogenesis? What are the targeted proteins of FBXO3 that are critically involved in tumor progression? What are other factors regulating the expression of $\mathrm{FBXO} 3$ in tumor cells? Is the high expression of FBXO3 associated with poor prognosis in various types of cancers? To find answers to these questions, FBXO3 knockout or knock-in transgenic mouse models could be used to validate the mechanism of FBXO3 in regulating the progression of human cancer. It is also important to look into various databases. Currently, the research on FBXO3 is still in its infancy, and further investigation is needed to develop better treatments using $\mathrm{FBXO} 3$ as a molecular target.

\section{AUTHOR CONTRIBUTIONS}

$\mathrm{ZZ}, \mathrm{ZB}, \mathrm{JY}$, and PG searched the literature and made the figures and tables. ZZ drafted the manuscript. PW and DC edited and revised the manuscript. All authors read and approved the final manuscript.

\section{FUNDING}

This work was supported by the Nature Science Major and Key Program of College and University of Anhui Province (No. KJ2020A0559 and KJ2021A0684), and 512 Talents Development Project of Bengbu Medical College (No. BY51201304).

Boshra, H., Lorenzo, G., Busquets, N., and Brun, A. (2011). Rift valley Fever: Recent Insights into Pathogenesis and Prevention. J. Virol. 85, 6098-6105. doi:10.1128/ jvi.02641-10

Bouloy, M., Janzen, C., Vialat, P., Khun, H., Pavlovic, J., Huerre, M., et al. (2001). Genetic Evidence for an Interferon-Antagonistic Function of Rift valley Fever Virus Nonstructural Protein NSs. J. Virol. 75, 1371-1377. doi:10.1128/ jvi.75.3.1371-1377.2001

Cha, J.-D., Kim, H. J., and Cha, I.-H. (2011). Genetic Alterations in Oral Squamous Cell Carcinoma Progression Detected by Combining Array-Based Comparative Genomic Hybridization and Multiplex Ligation-dependent Probe Amplification. Oral Surg. Oral Med. Oral Pathol. Oral Radiol. Endodontology 111, 594-607. doi:10.1016/j.tripleo.2010.11.020

Chai, S., Tong, M., Ng, K. Y., Kwan, P. S., Chan, Y. P., Fung, T. M., et al. (2014). Regulatory Role of miR-142-3p on the Functional Hepatic Cancer Stem Cell Marker CD133. Oncotarget 5, 5725-5735. doi:10.18632/oncotarget.2167

Chandra, D., Londino, J., Alexander, S., Bednash, J. S., Zhang, Y., Friedlander, R. M., et al. (2019). The SCFFBXO3 Ubiquitin E3 Ligase Regulates Inflammation in Atherosclerosis. J. Mol. Cell Cardiol. 126, 50-59. doi:10.1016/j.yjmcc.2018.11.006 
Chen, B. B., Coon, T. A., Glasser, J. R., McVerry, B. J., Zhao, J., Zhao, Y., et al. (2013). A Combinatorial F Box Protein Directed Pathway Controls TRAF Adaptor Stability to Regulate Inflammation. Nat. Immunol. 14, 470-479. doi:10.1038/ni.2565

Cheng, L., Tang, X., Xu, L., Zhang, L., Shi, H., Peng, Q., et al. (2019). Interferon- $\gamma$ Upregulates $\triangle 42 \mathrm{PD} 1$ Expression on Human Monocytes via the PI3K/AKT Pathway. Immunobiology 224, 388-396. doi:10.1016/j.imbio.2019.02.009

Chesarone, M. A., DuPage, A. G., and Goode, B. L. (2010). Unleashing Formins to Remodel the Actin and Microtubule Cytoskeletons. Nat. Rev. Mol. Cel Biol 11, 62-74. doi: $10.1038 / \mathrm{nrm} 2816$

Chia, R., Chiò, A., and Traynor, B. J. (2018). Novel Genes Associated with Amyotrophic Lateral Sclerosis: Diagnostic and Clinical Implications. Lancet Neurol. 17, 94-102. doi:10.1016/s1474-4422(17)30401-5

Coppola, T., Magnin-Lüthi, S., Perret-Menoud, V., Gattesco, S., Schiavo, G., and Regazzi, R. (2001). Direct Interaction of the Rab3 Effector RIM with Ca2+Channels, SNAP-25, and Synaptotagmin. J. Biol. Chem. 276, 32756-32762. doi:10.1074/jbc.m100929200

Daou, P., Hasan, S., Breitsprecher, D., Baudelet, E., Camoin, L., Audebert, S., et al. (2014). Essential and Nonredundant Roles for Diaphanous Formins in Cortical Microtubule Capture and Directed Cell Migration. MBoC 25, 658-668. doi:10.1091/mbc.e13-08-0482

de Thé, H., Chomienne, C., Lanotte, M., Degos, L., and Dejean, A. (1990). The $\mathrm{T}(15 ; 17)$ Translocation of Acute Promyelocytic Leukaemia Fuses the Retinoic Acid Receptor a Gene to a Novel Transcribed Locus. Nature 347, 558-561. doi:10.1038/347558a0

de Thé, H., Lavau, C., Marchio, A., Chomienne, C., Degos, L., and Dejean, A. (1991). The PML-Rara Fusion mRNA Generated by the T(15;17) Translocation in Acute Promyelocytic Leukemia Encodes a Functionally Altered RAR. Cell 66, 675-684. doi:10.1016/0092-8674(91)90113-d

Dinarello, C. A. (2007). Historical Insights into Cytokines. Eur. J. Immunol. 37 (Suppl. 1), S34-S45. doi:10.1002/eji.200737772

Dong, H., and Song, J. (2021). miR-142-3p Reduces the Viability of Human Cervical Cancer Cells by Negatively Regulating the Cytoplasmic Localization of HMGB1. Exp. Ther. Med. 21, 212. doi:10.3892/etm.2021.9644

Fang, X., Lin, H., Wang, X., Zuo, Q., Qin, J., and Zhang, P. (2015). The NEK1 Interactor, C21ORF2, Is Required for Efficient DNA Damage Repair. Acta Biochim. Biophys. Sin 47, 834-841. doi:10.1093/abbs/gmv076

Finnish-German, A. C. (1997). An Autoimmune Disease, APECED, Caused by Mutations in a Novel Gene Featuring Two PHD-type Zinc-finger Domains. Nat. Genet. 17, 399-403. doi:10.1038/ng1297-399

Fu, L., Cui, C.-P., Zhang, X., and Zhang, L. (2020). The Functions and Regulation of Smurfs in Cancers. Semin. Cancer Biol. 67, 102-116. doi:10.1016/j.semcancer.2019.12.023

Gavanescu, I., Kessler, B., Ploegh, H., Benoist, C., and Mathis, D. (2007). Loss of Aire-dependent Thymic Expression of a Peripheral Tissue Antigen Renders it a Target of Autoimmunity. Proc. Natl. Acad. Sci. 104, 4583-4587. doi:10.1073/ pnas.0700259104

Ghafouri-Fard, S., Hajiesmaeili, M., Shoorei, H., Bahroudi, Z., Taheri, M., and Sharifi, G. (2021). The Impact of lncRNAs and miRNAs in Regulation of Function of Cancer Stem Cells and Progression of Cancer. Front. Cel Dev. Biol. 9, 696820. doi:10.3389/fcell.2021.696820

Goddard, A. D., Borrow, J., Freemont, P. S., and Solomon, E. (1991). Characterization of a Zinc finger Gene Disrupted by the $\mathrm{T}(15 ; 17)$ in Acute Promyelocytic Leukemia. Science 254, 1371-1374. doi:10.1126/science.1720570

Goodall, G. J., and Wickramasinghe, V. O. (2021). RNA in Cancer. Nat. Rev. Cancer 21, 22-36. doi:10.1038/s41568-020-00306-0

Grabbe, C., Husnjak, K., and Dikic, I. (2011). The Spatial and Temporal Organization of Ubiquitin Networks. Nat. Rev. Mol. Cel Biol 12, 295-307. doi:10.1038/nrm3099

Han, S., Lear, T. B., Jerome, J. A., Rajbhandari, S., Snavely, C. A., Gulick, D. L., et al. (2015). Lipopolysaccharide Primes the NALP3 Inflammasome by Inhibiting its Ubiquitination and Degradation Mediated by the SCFFBXL2 E3 Ligase. J. Biol. Chem. 290, 18124-18133. doi:10.1074/jbc.m115.645549

Hao, Y., Baker, D., and Ten Dijke, P. (2019). TGF- $\beta$-Mediated EpithelialMesenchymal Transition and Cancer Metastasis. Int. J. Mol. Sci. 20. doi:10.3390/ijms20112767

Hernández-Ramírez, L. C., Martucci, F., Morgan, R. M. L., Trivellin, G., Tilley, D., Ramos-Guajardo, N., et al. (2016). Rapid Proteasomal Degradation of Mutant Proteins Is the Primary Mechanism Leading to Tumorigenesis in Patients with
MissenseAIPMutations. J. Clin. Endocrinol. Metab. 101, 3144-3154. doi:10.1210/jc.2016-1307

Hess-Stumpp, H. (2005). Histone Deacetylase Inhibitors and Cancer: from Cell Biology to the Clinic. Eur. J. Cel Biol. 84, 109-121. doi:10.1016/j.ejcb.2004.12.010

Hibino, H., Pironkova, R., Onwumere, O., Vologodskaia, M., Hudspeth, A. J., and Lesage, F. (2002). RIM Binding Proteins (RBPs) Couple Rab3-Interacting Molecules (RIMs) to Voltage-Gated Ca2+ Channels. Neuron 34, 411-423. doi:10.1016/s0896-6273(02)00667-0

Hung, K.-Y., Liao, W.-I., Pao, H.-P., Wu, S.-Y., Huang, K.-L., and Chu, S.-J. (2019). Targeting F-Box Protein Fbxo3 Attenuates Lung Injury Induced by IschemiaReperfusion in Rats. Front. Pharmacol. 10, 583. doi:10.3389/fphar.2019.00583

Hussain, N. K., Hsin, H., Huganir, R. L., and Sheng, M. (2010). MINK and TNIK Differentially Act on Rap2-Mediated Signal Transduction to Regulate Neuronal Structure and AMPA Receptor Function. J. Neurosci. 30, 14786-14794. doi:10.1523/jneurosci.4124-10.2010

Inoue, J.-i., Ishida, T., Tsukamoto, N., Kobayashi, N., Naito, A., Azuma, S., et al. (2000). Tumor Necrosis Factor Receptor-Associated Factor (TRAF) Family: Adapter Proteins that Mediate Cytokine Signaling. Exp. Cel Res. 254, 14-24. doi:10.1006/excr.1999.4733

Isobe, T., Hisamori, S., Hogan, D. J., Zabala, M., Hendrickson, D. G., Dalerba, P., et al. (2014). miR-142 Regulates the Tumorigenicity of Human Breast Cancer Stem Cells through the Canonical WNT Signaling Pathway. Elife 3. doi:10.7554/eLife.01977

Isogai, T., van der Kammen, R., Goerdayal, S. S., Heck, A. R., Altelaar, A. F. M., and Innocenti, M. (2015). Proteomic Analyses Uncover a New Function and Mode of Action for Mouse Homolog of Diaphanous $2(\mathrm{mDia} 2)^{*}$. Mol. Cell Proteomics 14, 1064-1078. doi:10.1074/mcp.m114.043885

Jiang, W., Xia, J., Xie, S., Zou, R., Pan, S., Wang, Z.-w., et al. (2020). Long Noncoding RNAs as a Determinant of Cancer Drug Resistance: Towards the Overcoming of Chemoresistance via Modulation of lncRNAs. Drug Resist. Updates 50, 100683. doi:10.1016/j.drup.2020.100683

Jin, Y., Mailloux, C. M., Gowan, K., Riccardi, S. L., LaBerge, G., Bennett, D. C., et al. (2007). NALP1in Vitiligo-Associated Multiple Autoimmune Disease. N. Engl. J. Med. 356, 1216-1225. doi:10.1056/nejmoa061592

Kainulainen, M., Habjan, M., Hubel, P., Busch, L., Lau, S., Colinge, J., et al. (2014). Virulence Factor NSs of Rift valley Fever Virus Recruits the F-Box Protein FBXO3 to Degrade Subunit P62 of General Transcription Factor TFIIH. J. Virol. 88, 3464-3473. doi:10.1128/jvi.02914-13

Kakizuka, A., Miller, W. H., Jr., Umesono, K., Warrell, R. P., Jr., Frankel, S. R., Murty, V. V. V. S., et al. (1991). Chromosomal Translocation T(15;17) in Human Acute Promyelocytic Leukemia Fuses RARa with a Novel Putative Transcription Factor, PML. Cell 66, 663-674. doi:10.1016/0092-8674(91)90112-c

Kirsten, K., Fior, D., Kreutz, L. C., and Barcellos, L. J. G. (2018). First Description of Behavior and Immune System Relationship in Fish. Sci. Rep. 8, 846. doi:10.1038/s41598-018-19276-3

Kong, L. R., Tan, T. Z., Ong, W. R., Bi, C., Huynh, H., Lee, S. C., et al. (2017). Belinostat Exerts Antitumor Cytotoxicity through the Ubiquitin-Proteasome Pathway in Lung Squamous Cell Carcinoma. Mol. Oncol. 11, 965-980. doi:10.1002/1878-0261.12064

Krzysiak, T. C., Chen, B. B., Lear, T., Mallampalli, R. K., and Gronenborn, A. M. (2016). Crystal Structure and Interaction Studies of the Human FBxo3 ApaG Domain. FEBS J. 283, 2091-2101. doi:10.1111/febs.13721

Lai, C.-Y., Ho, Y.-C., Hsieh, M.-C., Wang, H.-H., Cheng, J.-K., Chau, Y.-P., et al. (2016). Spinal Fbxo3-dependent Fbxl2 Ubiquitination of Active Zone Protein RIM1 Mediates Neuropathic Allodynia through CaV2.2 Activation. J. Neurosci. 36, 9722-9738. doi:10.1523/jneurosci.1732-16.2016

Li, D., Xie, P., Zhao, F., Shu, J., Li, L., Zhan, Y., et al. (2015). F-box Protein Fbxo3 Targets Smurf1 Ubiquitin Ligase for Ubiquitination and Degradation. Biochem. Biophysical Res. Commun. 458, 941-945. doi:10.1016/j.bbrc.2015.02.089

Li, J., and Ma, L. (2020). MiR-142-3p Attenuates Oxygen Glucose Deprivation/ Reoxygenation-Induced Injury by Targeting FBXO3 in Human Neuroblastoma SH-Sy5y Cells. World Neurosurg. 136, e149-e157. doi:10.1016/j.wneu.2019.12.064

Li, Z., Fan, S., Wang, J., Chen, X., Liao, Q., Liu, X., et al. (2020). Zebrafish F-Box Protein Fbxo3 Negatively Regulates Antiviral Response through Promoting K27-Linked Polyubiquitination of the Transcription Factors Irf3 and Irf7. J.I. 205, 1897-1908. doi:10.4049/jimmunol.2000305

Lin, M., Xu, Y., Gao, Y., Pan, C., Zhu, X., and Wang, Z.-w. (2019). Regulation of F-Box Proteins by Noncoding RNAs in Human Cancers. Cancer Lett. 466, 61-70. doi:10.1016/j.canlet.2019.09.008 
Lin, T.-B., Hsieh, M.-C., Lai, C.-Y., Cheng, J.-K., Chau, Y.-P., Ruan, T., et al. (2015). Fbxo3-Dependent Fbxl2 Ubiquitination Mediates Neuropathic Allodynia through the TRAF2/TNIK/GluR1 Cascade. J. Neurosci. 35, 16545-16560. doi:10.1523/jneurosci.2301-15.2015

Lizárraga, F., Poincloux, R., Romao, M., Montagnac, G., Le Dez, G., Bonne, I., et al. (2009). Diaphanous-related Formins Are Required for Invadopodia Formation and Invasion of Breast Tumor Cells. Cancer Res. 69, 2792-2800. doi:10.1158/ 0008-5472.can-08-3709

Lu, L.-F., Li, S., Wang, Z.-X., Liu, S.-B., Chen, D.-D., and Zhang, Y.-A. (2019). Zebrafish NDRG1a Negatively Regulates IFN Induction by Promoting the Degradation of IRF7. J.I. 202, 119-130. doi:10.4049/jimmunol.1800490

Mallampalli, R. K., Coon, T. A., Glasser, J. R., Wang, C., Dunn, S. R., Weathington, N. M., et al. (2013). Targeting F Box Protein Fbxo3 to Control Cytokine-Driven Inflammation. J.I. 191, 5247-5255. doi:10.4049/jimmunol.1300456

Man, S. M., and Kanneganti, T.-D. (2015). Regulation of Inflammasome Activation. Immunol. Rev. 265, 6-21. doi:10.1111/imr.12296

Masuda, K., Masuda, R., Neidhart, M., Simmen, B. R., Michel, B. A., MüllerLadner, U., et al. (2002). Molecular Profile of Synovial Fibroblasts in Rheumatoid Arthritis Depends on the Stage of Proliferation. Arthritis Res. 4, R8. doi:10.1186/ar427

Minucci, S., and Pelicci, P. G. (2006). Histone Deacetylase Inhibitors and the Promise of Epigenetic (And More) Treatments for Cancer. Nat. Rev. Cancer 6, 38-51. doi:10.1038/nrc1779

Nagamine, K., Peterson, P., Scott, H. S., Kudoh, J., Minoshima, S., Heino, M., et al. (1997). Positional Cloning of the APECED Gene. Nat. Genet. 17, 393-398. doi:10.1038/ng1297-393

Nathan, C. (2002). Points of Control in Inflammation. Nature 420, 846-852. doi:10.1038/nature01320

Niu, M., He, Y., Xu, J., Ding, L., He, T., Yi, Y., et al. (2021). Noncanonical TGF- $\beta$ Signaling Leads to FBXO3-Mediated Degradation of $\triangle$ Np63a Promoting Breast Cancer Metastasis and Poor Clinical Prognosis. Plos Biol. 19, e3001113. doi:10.1371/journal.pbio.3001113

Palumbo, A., Chanan-Khan, A., Weisel, K., Nooka, A. K., Masszi, T., Beksac, M., et al. (2016). Daratumumab, Bortezomib, and Dexamethasone for Multiple Myeloma. N. Engl. J. Med. 375, 754-766. doi:10.1056/nejmoa1606038

Pantzaris, N. D., Platanaki, C., Tsiotsios, K., Koniari, I., and Velissaris, D. (2021). The Use of Electroencephalography in Patients with Sepsis: a Review of the Literature. J. Transl Int. Med. 9, 12-16. doi:10.2478/jtim-2021-0007

Perheentupa, J. (2006). Autoimmune Polyendocrinopathy-Candidiasis-Ectodermal Dystrophy. J. Clin. Endocrinol. Metab. 91, 2843-2850. doi:10.1210/jc.2005-2611

Peterson, P., Org, T., and Rebane, A. (2008). Transcriptional Regulation by AIRE: Molecular Mechanisms of central Tolerance. Nat. Rev. Immunol. 8, 948-957. doi:10.1038/nri2450

Peterson, P., and Peltonen, L. (2005). Autoimmune Polyendocrinopathy Syndrome Type 1 (APS1) and AIRE Gene: New Views on Molecular Basis of Autoimmunity. J. Autoimmun. 25 (Suppl. l), 49-55. doi:10.1016/j.jaut.2005.09.022

Popovic, D., Vucic, D., and Dikic, I. (2014). Ubiquitination in Disease Pathogenesis and Treatment. Nat. Med. 20, 1242-1253. doi:10.1038/nm.3739

Sabater, L., Ferrer-Francesch, X., Sospedra, M., Caro, P., Juan, M., and PujolBorrell, R. (2005). Insulin Alleles and Autoimmune Regulator (AIRE) Gene Expression Both Influence Insulin Expression in the Thymus. J. Autoimmun. 25, 312-318. doi:10.1016/j.jaut.2005.08.006

Saegusa, H., and Tanabe, T. (2014). N-type Voltage-dependent Ca2+ Channel in Non-excitable Microglial Cells in Mice Is Involved in the Pathophysiology of Neuropathic Pain. Biochem. Biophysical Res. Commun. 450, 142-147. doi:10.1016/j.bbrc.2014.05.103

Schoch, S., Mittelstaedt, T., Kaeser, P. S., Padgett, D., Feldmann, N., Chevaleyre, V., et al. (2006). Redundant Functions of RIMl $\alpha$ and RIM2 $\alpha$ in Ca2+-Triggered Neurotransmitter Release. EMBO J. 25, 5852-5863. doi:10.1038/sj.emboj.7601425

Schroder, K., and Tschopp, J. (2010). The Inflammasomes. Cell 140, 821-832. doi:10.1016/j.cell.2010.01.040

Senft, D., Qi, J., and Ronai, Z. e. A. (2018). Ubiquitin Ligases in Oncogenic Transformation and Cancer Therapy. Nat. Rev. Cancer 18, 69-88. doi:10.1038/ nrc.2017.105

Shao, W., Zumer, K., Fujinaga, K., and Peterlin, B. M. (2016). FBXO3 Protein Promotes Ubiquitylation and Transcriptional Activity of AIRE (Autoimmune Regulator). J. Biol. Chem. 291, 17953-17963. doi:10.1074/jbc.m116.724401
Sheu, C.-C., Gong, M. N., Zhai, R., Bajwa, E. K., Chen, F., Taylor Thompson, B., et al. (2010). The Influence of Infection Sites on Development and Mortality of ARDS. Intensive Care Med. 36, 963-970. doi:10.1007/s00134-010-1851-3

Shima, Y., Shima, T., Chiba, T., Irimura, T., Pandolfi, P. P., and Kitabayashi, I. (2008). PML Activates Transcription by Protecting HIPK2 and P300 from SCF Fbx3 -Mediated Degradation. Mol. Cel Biol 28, 7126-7138. doi:10.1128/ mcb.00897-08

Shrestha, A., Mukhametshina, R. T., Taghizadeh, S., Vásquez-Pacheco, E., CabreraFuentes, H., Rizvanov, A., et al. (2017). MicroRNA-142is a Multifaceted Regulator in Organogenesis, Homeostasis, and Disease. Dev. Dyn. 246, 285-290. doi:10.1002/dvdy.24477

Skaar, J. R., Pagan, J. K., and Pagano, M. (2013). Mechanisms and Function of Substrate Recruitment by F-Box Proteins. Nat. Rev. Mol. Cel Biol 14, 369-381. doi:10.1038/nrm3582

Skaar, J. R., Pagan, J. K., and Pagano, M. (2014). SCF Ubiquitin Ligase-Targeted Therapies. Nat. Rev. Drug Discov. 13, 889-903. doi:10.1038/nrd4432

Song, Y., Lin, M., Liu, Y., Wang, Z.-W., and Zhu, X. (2019). Emerging Role of F-Box Proteins in the Regulation of Epithelial-Mesenchymal Transition and Stem Cells in Human Cancers. Stem Cel Res Ther 10, 124. doi:10.1186/s13287-0191222-0

Talbott, E. O., Malek, A. M., and Lacomis, D. (2016). The Epidemiology of Amyotrophic Lateral Sclerosis. Handb Clin. Neurol. 138, 225-238. doi:10.1016/b978-0-12-802973-2.00013-6

Wang, Q., Chen, W., and Lin, J. (2019). The Role of Calprotectin in Rheumatoid Arthritis. J. Transl Int. Med. 7, 126-131. doi:10.2478/jtim-2019-0026

Wang, Z.-w., Hu, X., Ye, M., Lin, M., Chu, M., and Shen, X. (2020). NEDD4 E3 Ligase: Functions and Mechanism in Human Cancer. Semin. Cancer Biol. 67, 92-101. doi:10.1016/j.semcancer.2020.03.006

Wang, Z., Liu, P., Inuzuka, H., and Wei, W. (2014). Roles of F-Box Proteins in Cancer. Nat. Rev. Cancer 14, 233-247. doi:10.1038/nrc3700

Watanabe, Y., Nakagawa, T., Akiyama, T., Nakagawa, M., Suzuki, N., Warita, H., et al. (2020). An Amyotrophic Lateral Sclerosis-Associated Mutant of C21ORF2 Is Stabilized by NEK1-Mediated Hyperphosphorylation and the Inability to Bind FBXO3. iScience 23, 101491. doi:10.1016/j.isci.2020.101491

Winkle, M., El-Daly, S. M., Fabbri, M., and Calin, G. A. (2021). Noncoding RNA Therapeutics - Challenges and Potential Solutions. Nat. Rev. Drug Discov. 20, 629-651. doi:10.1038/s41573-021-00219-z

Winston, J. T., Koepp, D. M., Zhu, C., Elledge, S. J., and Harper, J. W. (1999). A Family of Mammalian F-Box Proteins. Curr. Biol. 9, 1180-S3. doi:10.1016/ s0960-9822(00)80021-4

Xiao, P., and Liu, W. L. (2015). MiR-142-3p Functions as a Potential Tumor Suppressor Directly Targeting HMGB1 in Non-small-cell Lung Carcinoma. Int. J. Clin. Exp. Pathol. 8, 10800-10807.

Xu, T., He, B. S., Pan, B., Pan, Y. Q., Sun, H. L., Liu, X. X., et al. (2020). MiR-142-3p Functions as a Tumor Suppressor by Targeting RAC1/PAK1 Pathway in Breast Cancer. J. Cel Physiol 235, 4928-4940. doi:10.1002/jcp.29372

Yan, L., Lin, M., Pan, S., Assaraf, Y. G., Wang, Z.-w., and Zhu, X. (2020). Emerging Roles of F-Box Proteins in Cancer Drug Resistance. Drug Resist. Updates 49, 100673. doi:10.1016/j.drup.2019.100673

Conflict of Interest: The authors declare that the research was conducted in the absence of any commercial or financial relationships that could be construed as a potential conflict of interest.

Publisher's Note: All claims expressed in this article are solely those of the authors and do not necessarily represent those of their affiliated organizations, or those of the publisher, the editors, and the reviewers. Any product that may be evaluated in this article, or claim that may be made by its manufacturer, is not guaranteed or endorsed by the publisher.

Copyright $\odot 2022$ Zhang, Bao, Gao, Yao, Wang and Chai. This is an open-access article distributed under the terms of the Creative Commons Attribution License (CC BY). The use, distribution or reproduction in other forums is permitted, provided the original author(s) and the copyright owner(s) are credited and that the original publication in this journal is cited, in accordance with accepted academic practice. No use, distribution or reproduction is permitted which does not comply with these terms. 